\title{
Front2Back: Single View 3D Shape Reconstruction via Front to Back Prediction
}

\author{
Yuan Yao ${ }^{1}$ Nico Schertler ${ }^{1}$ Enrique Rosales ${ }^{1,2}$ Helge Rhodin $^{1}$ Leonid Sigal ${ }^{1,3}$ Alla Sheffer ${ }^{1}$ \\ ${ }^{1}$ University of British Columbia \\ ${ }^{2}$ Universidad Panamericana \\ ${ }^{3}$ Canada CIFAR AI Chair, Vector Institute \\ \{rozentil, nschertl, albertr, rhodin, lsigal, sheffa\}ecs.ubc.ca
}

\begin{abstract}
Reconstruction of a $3 D$ shape from a single $2 D$ image is a classical computer vision problem, whose difficulty stems from the inherent ambiguity of recovering occluded or only partially observed surfaces. Recent methods address this challenge through the use of largely unstructured neural networks that effectively distill conditional mapping and priors over $3 D$ shape.

In this work, we induce structure and geometric constraints by leveraging three core observations: (1) the surface of most everyday objects is often almost entirely exposed from pairs of typical opposite views; (2) everyday objects often exhibit global reflective symmetries which can be accurately predicted from single views; (3) opposite orthographic views of a $3 D$ shape share consistent silhouettes.
\end{abstract}

Following these observations, we first predict orthographic $2.5 D$ visible surface maps (depth, normal and silhouette) from perspective $2 D$ images, and detect global reflective symmetries in this data; second, we predict the back facing depth and normal maps using as input the front maps and, when available, the symmetric reflections of these maps; and finally, we reconstruct a $3 D$ mesh from the union of these maps using a surface reconstruction method best suited for this data.

Our experiments demonstrate that our framework outperforms state-of-the art approaches for $3 D$ shape reconstructions from $2 D$ and $2.5 D$ data in terms of input fidelity and details preservation. Specifically, we achieve $12 \%$ better performance on average in ShapeNet benchmark dataset [3], and up to $19 \%$ for certain classes of objects (e.g., chairs and vessels).

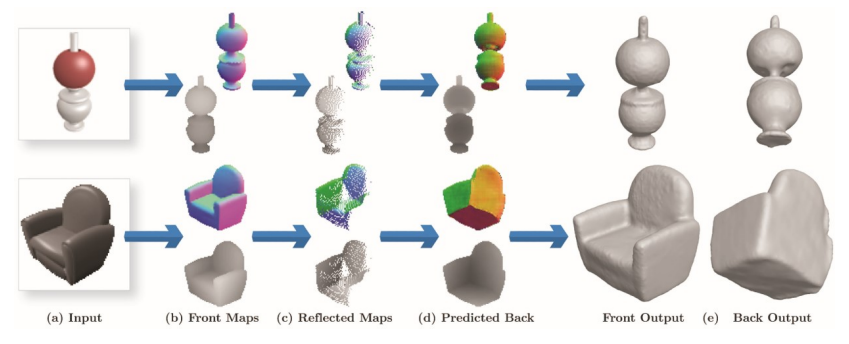

Figure 1: Front2Back reconstructs 3D surfaces (left) from single $2 \mathrm{D}$ images (a) by first computing the corresponding front facing 2.5D normal, depth and silhouette (not shown) visible surface maps (b) and their symmetric reflections (when present) (c) ; it then predicts the back (opposite) view maps (d); and finally fuses all these intermediate maps via surface reconstruction to produce a watertigth $3 \mathrm{D}$ surface (e). Our approach is able to recover more detailed and faithful 3D shape reconstructions compared to state-of-the-art.

\section{Introduction}

Humans are amazingly adept at predicting the shape of $3 \mathrm{D}$ objects from a single 2D image, a task made particularly challenging by the need to predict occluded surface geometry. Early computer vision researchers hypothesized that humans achieve this goal by employing a series of intermediate representations, of progressively increasing complexity, and envision the $2.5 \mathrm{D}$ visible surface geometry as one of these representations [26]. This argument motivates multi-step 3D reconstruction pipelines that use $2.5 \mathrm{D}$ visible surface as an intermediate representation [46]. Our novel Front2Back algorithm targets complete 3D surface reconstruction from such an intermediate representation, encoded via multiple depth and normal maps that capture global reflective symmetries (Figure $1 \mathrm{bc}$ ). We create a complete image to 3D reconstruction framework by combining Front2Back with a data-driven predictor that computes ac- 
curate $2.5 \mathrm{D}$ visible surface geometry from raw $2 \mathrm{D}$ images undoing perspective effects (Figure 1a).

Recent methods directly learn 3D shape representation from raw 2D images $[6,7,16,27,37,43]$ or intermediate $2.5 \mathrm{D}$ visible surface maps $[35,42,44,46]$ and represent 3D shapes using voxelizations or implicit functions (defined over $\mathbb{R}^{3}$ ). Such models often implicitly distill shape prior information into the network through a large set of 2D-3D paired exemplars obtained from ShapeNet [3] or similar large scale 3D model repository. These methods, collectively, produced an increasingly impressive set of results on single-view 3D shape reconstruction, but are limited in terms of fidelity, a limitation which can be largely attributed to the resolution of the resulting voxel grid, as well as to the inherent complexity of learning volumeteric $\left(\mathbb{R}^{3}\right)$ information directly from images $\left(\mathbb{R}^{2}\right)$. While the dimensionality challenge can be sidestepped via the use of $2 \mathrm{D}$ (texture) atlases describing the output point-clouds [16] or the use of deformable templates [38, 41], these approaches face other challenges. Notably, none of the existing methods take explicit advantage of geometric cues correlating visible and occluded surface parts; nor leverage strong perceptual cues in the input image, such as symmetry, which has been shown to be integral for the human 3D perception [31].

Similar to [16] we utilize an image-centric 2.5D intermediate data representation which allows us to describe output point-clouds precisely. However, contrary to all the above approaches we explicitly leverage geometric and perceptual linkages between visible and occluded surface geometry. The core observation behind our framework is that most everyday objects can be almost entirely described by pairs of oppositely oriented height-fields; moreover this property holds for most orientation choices ${ }^{1}$. Therefore, a pair of $2.5 \mathrm{D}$ visible surface images taken from opposite views frequently describes a (nearly) complete 3D model (Figure 1b, 1c). Notably for orthographic projection, the silhouettes of such opposite images always align. Consequently, the problem of reconstructing 3D models from single, non-accidental, 2.5 orthographic visible surface images can for many classes of objects be effectively reduced to the Front2Back problem: correctly recovering the back facing $2.5 \mathrm{D}$ visible surface given a front facing one. The core advantage of this approach is that instead of directly obtaining $3 \mathrm{D}$ data from a $2 \mathrm{D}$ or a $2.5 \mathrm{D}$ input, a process that is still not well understood, we can now recast our core problem as synthesizing one type of, silhouette aligned, image (back) from another (front), a task that had been successfully tackled by recent image-to-image translation networks. Lastly, and critically, many everyday shapes exhibit global reflec-

\footnotetext{
${ }^{1}$ In our experiments, a random sample of 520 shapes from ShapeNet core had on average $80 \%$ of their surface visible from $95 \%$ of random opposite view pairs.
}

tive symmetries, which can be accurately detected from front visible surface maps alone. These symmetries allow viewers to predict backfacing geometry as well as to complete details not available in either front or back views.

Following these observations, Front2Back employs an image-to-image translation network to predict back view $2.5 \mathrm{D}$ visible surface maps (depth+normal) from the input front $2.5 \mathrm{D}$ representation. It then reconstructs a 3D surface mesh fusing together the front and back geometry information using a reconstruction framework capable of producing watertight surfaces by combining positional and normal information [22]. In both the prediction of the intermediate $2.5 \mathrm{D}$ back view maps and the reconstruction itself, it leverages global reflective symmetries, algorithmically detected on the front visible surfaces, when present: reflected front surface maps (Figure 1) are provided as auxiliary input for back prediction, and used as an additional source of data for subsequent reconstruction. To achieve direct 3D reconstruction from raw images we combine our method with a learning based framework that for each input image computes the corresponding orthographic $2.5 \mathrm{D}$ visible surfaces rectifying perspective distortion present in these images. Our approach leads to significant improvements in the resulting 3D shape reconstruction, compared to competing state-of-the-art methods, resulting in $12 \%$ improvements on average, measured using mesh-to-mesh distance [8] and up to $19 \%$ improvements on certain ShapeNet object classes, compared to the closest competitors.

Contribution: Our core contribution is a novel framework for $2.5 \mathrm{D}$ visible surface map to $3 \mathrm{D}$ reconstruction, which combines recent learning-based approaches with more traditional geometric processing and produces results superior to the state-of-the-art. This contribution is made possible by two core technical innovations: (1) novel and effective intermediate representation consisting of the orthographic 2.5D back view of the object, which we obtain using a stateof-the-art image-to-image translation architecture; and (2) utilization of symmetries detected in the front view to significantly improve the performance of both the $2.5 \mathrm{D}$ back view prediction and overall surface reconstruction.

\section{Related Work}

We build upon a large body of work on single-view 3D surface reconstruction, view synthesis, and image-to-image translation.

Single-view 3D Surface Reconstruction. Reconstructing 3D models from single view $2 \mathrm{D}$ images, or $2.5 \mathrm{D}$ depth (and normal) maps, is a difficult and ill-posed problem. Recent learning based methods show promising results in addressing this challenge [36]. While many methods predict 3D shape representations directly from $2 \mathrm{D}$ images, e.g., $[6,7,11,13,16,27,37,38,43,45]$, others, 
e.g., $[35,42,44]$ first reconstruct the $2.5 \mathrm{D}$ visible surface (typically representing it via depth and normal maps) and then use this intermediate representation as a stepping stone toward complete 3D reconstruction. Many methods in both categories represent the reconstructed shapes using voxels, e.g., [13, 42, 44, 45] or limited-depth octrees [39]. The accuracy of the reconstructions produced by these methods is limited by the finite resolution of the voxel or octree cells, limiting the methods' ability to capture fine details. Template based methods [34, 38, 41] perform well when the topology of the input template matches that of the depicted shape, but are less suited for cases where the target topology is not known a priori. Recent implicit surface based methods $[6,27]$ strive for resolution independence but require watertight meshes for training. Since the vast majority of meshes in the wild are far from watertight ${ }^{2}$ instead of training directly on this data they use watertight approximations which necessarily deviate from the originals. This deviation can potentially bias surface prediction. Atlas based [16] reconstruction avoids these pitfalls, but exhibits similar accuracy levels. Point or depth-only maps based methods [11, 33, 24] produce collections of points close to the target objects surface; however surface reconstruction from unoriented points is a challenging problem in its own right [2], thus when attempting to recover the surface from cloud the output quality decreases dramatically [33]. Our method uses depth plus normal maps as intermediate representation, works well on shapes with diverse topology, has no bounds on accuracy beyond the input image resolution; and can be directly trained on models with arbitrary connectivity and non-manifold artifacts, avoiding the bias introduced by implicit function based approaches.

Two recent methods reconstruct front and back depth map images of human subjects from photographs [12, 29]. Our method differs from those in utilizing a normal map alongside depth, symmetric cues, and normalized, othographic coordinates obtained through perspective correction to predict depth maps across a wide range of classes/views.

Symmetries are used in [41], but only for regularizing the output at training time, unlike our explicit integration of symmetry as a cue for reconstruction, which requires symmetry detection on the input image at test time.

View Synthesis and Shape Completion. Our core task of predicting back view $2.5 \mathrm{D}$ surface can be seen as a special case of alternative view synthesis [9, 10, 15, 25, 47]. Most such methods aim to predict views that have similar viewpoints to the original. Recent approaches, [5] render compelling images and depth maps from viewpoints that differ by up to $40^{\circ}$ from the original. Surface reconstruction from depth maps alone $[24,33]$ suffers from similar pitfalls as one from unoriented clouds [2]. In contrast to these settings

\footnotetext{
${ }^{2}$ Less than $2.5 \%$ of the $7 \mathrm{~K}+$ models in the test split of ShapeNet core are watertight.
}

we seek to recover the exact opposite view, where the only overlap between the observed surfaces is along the outer silhouette. By combining these strategically selected views and utilizing symmetry we successfully compute oriented point-clouds, allowing for the use of more robust reconstruction methods [22] and producing far superior reconstruction results.

Learning based shape completion methods, e.g., [17, 30], attempt to extend partial surfaces across poorly captured regions to produce a complete shape representation. These methods are designed to operate on 3D shape inputs, are typically limited to filling relatively small holes, and employ shape priors for ambiguity resolution. In contrast, our Front2Back step predicts a complete surface from only front facing depth+normal maps and uses an intermediate $2.5 \mathrm{D}$ back map to achieve this goal.

Image-to-image translation. Image-to-image translation $[20,21,23,40,48]$ is a powerful tool for synthesizing new images from existing ones for applications such as imagefrom-sketch synthesis [32] and make-up application [4]. While typical translation methods aim to preserve the view and the content of the original image and only change some of their visual attributes, generating back views from front ones requires significant changes in the depth and normal content, a much more challenging task.

\section{Approach}

Our method takes as input a single perspective 2D image and generates a 3D mesh of the corresponding object, using four key steps (Figure 2). We start the process by predicting orthographic silhouette, depth, and normal maps of the portion of the target object's surface visible in the input image (Section 3.1). We proceed to locate a global 3D reflective symmetry plane from this visible surface, if one exists (Section 3.2). We use the located plane (if detected) to infer the occluded parts of the shape whose symmetric counterparts are visible, by reflecting the input maps over the symmetry plane to get a second set of depth and normal maps (hereon referred to as reflected maps). We mask all depth and normal maps using the silhuette, denoting all pixels outside as background, and use these maps an input to our core back prediction stage. The prediction stage takes this input and generates new depth and normal maps for the back view, the exact opposite of the input front view (Section 3.3). We perform this prediction using a variant of conditional generative adversarial networks for image to image translation. Finally, we combine the front view maps, reflected maps, and predicted back view maps to extract the corresponding oriented point cloud and reconstruct the surface from this cloud (see Section 3.4 for details). 


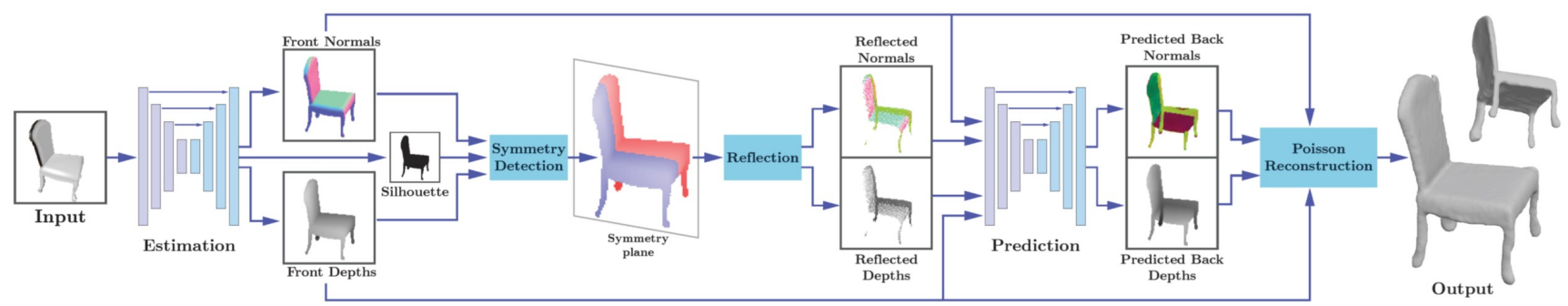

Figure 2: Algorithm Stages: (left to right): input perspective image; 2.5D orthographic map (depth+normal+silhouette) prediction; detection of reflective symmetries (regions on the left and right of the plane colored blue and red respectively); back map prediction; final surface reconstruction.

\subsection{Orthographic Front View Prediction}

For the $2 \mathrm{D}$ to $2.5 \mathrm{D}$ step, we adopt and train the $2.5 \mathrm{D}$ estimation network of [44], using example input-output pairs of perspective images and corresponding same view direction orthographic depth, normal, and silhouette maps. Perspective rectification simplifies subsequent symmetry estimation and allows us to enforce the same silhouette constraint across all computed maps.

We define the loss function as the sum of the three individual $L_{1}$ losses of the outputs. The original network is designed for noisy, real-life images and purposefully adds noise to input data to mimic real-life artifacts; since similar to most recent single view reconstruction methods our training image set is synthetic, for a fair comparison we disabled this feature.

\subsection{Symmetry Detection}

Reflective symmetry is a frequent feature of both organic and man made shapes. It plays a vital role in human perception - absent information to the contrary, human observers expect symmetries observed in the visible parts of a surface to extend into occluded parts facilitating mental reconstruction of these occluded surfaces [19]. Our method mimics this behavior by explicitly formulating a surface hypothesis derived from reflective symmetries detected on the front view maps. Efficient, robust, and accurate detection of reflective symmetry planes from partial surfaces is a challenging geometry processing problem $[1,28]$. While existing methods are designed for dense point-clouds, we seek to detect symmetries on pixelated and thus heavily quantized data, which frequently has very low local resolution (e.g. chair legs which are less than 5 pixels wide). Most critically, we seek to avoid false positives, as inaccurate reflected maps can severely impact our subsequent back prediction and reconstruction steps.

We design a targeted two-step reflective symmetry plane detection method, that addresses these challenges by combining information across all three front maps. We first estimate an approximate symmetry plane using a clustering- based approach, sped up using a variant of RANSAC. We refer to this plane as initial plane. We then optimize this initial plane to better align the original oriented points with their reflected counterparts using an iterated closest point method (Figure 3). We avoid false positives in both steps by usilizing two key constraints. We note that the silhouette map defines the visual hull of the target object, thus we expect any surface parts symmetric to parts of the visible surface to lie inside it; we thus filter out reflection planes that produce maps violating this constraint. Since our front maps are expected to be at least as accurate as the reflected ones, we similarly only consider planes that produce reflected maps that do not occlude the front surfaces (i.e. have no points closer to the viewer at the same x-y locations), we refer to this constraint as visibility. We use the detected planes to generate reflected depth and normal maps.

Initial plane. We use a clustering-based symmetry detection algorithm, inspired by [28] for estimating the initial symmetry plane. We first transform the front-facing normal and depth map into an oriented point set $P$. For each pair of oriented points $\left(\mathbf{p}_{i}, \mathbf{p}_{j}\right) \in P \times P$, we calculate the plane that best reflects $\mathbf{p}_{i}$ to $\mathbf{p}_{j}$. We then repeatedly sample subsets of such potential symmetry planes and cluster each subset using mean-shift clustering. We compute a Voronoi diagram of all planes with respect to the centers of the resulting clusters, and define the score of each center as the number of planes in its' respective cell. To select the initial plane among cluster centers obtained across all iterations we first discard all centers that produce reflected maps significantly violating visual hull or visibility constraints. We then select as initial plane the center with the highest score.

Optimization. Our initial plane is based on a sampling approach and only considers planes defined directly by point pairs, thus while close to the optimal, it can often be further improved. We optimize the plane using a variant of classical ICP. At each iteration, for each point $\mathbf{p} \in P$, we first calculate the point $\mathbf{r}_{p} \in P$ that is closest to its reflection around the current symmetry plane $s$. We prune all 

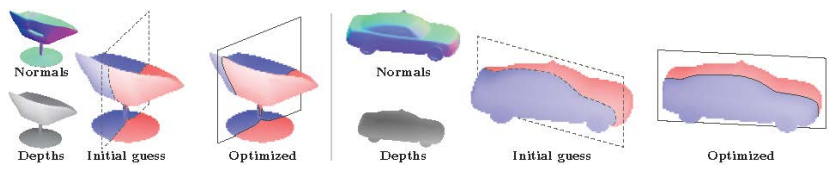

Figure 3: Symmetry plane detection. Given front view maps (insets, left) we use plane-space clustering to obtain initial reflective planes (center, dashed) and obtain the final planes (solid,right) by using ICP iterations. (For many inputs the improvement is more subtle.)

correspondences whose distance exceeds a user-definable threshold or whose normals are not reflected within a tolerance, and use the remaining correspondences $P_{c} \subseteq P$ to optimize for a symmetry plane that maps the points closer to their reflections, i.e.,

$$
s^{\prime}=\underset{s}{\arg \min } \sum_{\mathbf{p} \in P_{c}}\left\|\rho_{s}(\mathbf{p})-\mathbf{r}_{p}\right\|^{2} .
$$

We solve this minimization problem using gradient descent with back tracking line search to determine the step size and update the estimate for the symmetry plane with $s^{\prime}$. We repeat the process until convergence (Figure 3 , right).

Determining Model Symmetry. We classify the input as asymmetric if the resulting reflected maps violate visual hull or visibility constraints with tight thresholds, or generates a map which covers less than $40 \%$ of the silhouette interior. False symmetry can dramatically impact reconstruction quality, far more so than reconstruction with no symmetry information, motivating us to use fairly strict thresholds.In practice, we classify about $\approx 20 \%$ of the data as asymmetric.

\subsection{Back View Maps Prediction}

Recent deep learning-based image-to-image translation methods focus on the translation between different domains. In our method, we demonstrate that such deep neural networks can also be used to learn the mapping between $2.5 \mathrm{D}$ representations in opposing views.

Our learning model is similar to [21], which is based on a conditional generative adversarial network (cGAN). The architecture consists of two networks: a generator and a discriminator. The discriminator is trained on a training data set to classify an input image into either real or fake w.r.t. to training data set. The generator is trained to produce images that the discriminator evaluates as real. Our goal is to predict the back view normal and depth map from front view maps. To integrate symmetry information, we also feed the reflected depth and normal maps into the network when symmetries are detected (see end of Section 3.2). Hence the input to the generator is a depth- 4 or -8 image, depending on symmetry circumstance, and the output is a depth- 4 image encoding predicted back depth and normal maps. To train the network, we use a loss function $L$ that comprises terms for each of the individual aspects of our prediction problem:

$$
L=w_{G A N} L_{G A N}+w_{d} L_{d}+w_{n} L_{n} .
$$

The GAN loss $L_{G A N}$ is the traditional loss used for generative adversarial networks that steers the interplay between generator and discriminator. The two similarity loss functions for depth $L_{d}$ and normals $L_{n}$ aim to measure the pixelwise differences between predicted and ground truth maps. In our experiments, we set $w_{G A N}=1, w_{n}=100$, and $w_{d}=1000$. In the following, we present the loss functions in more detail.

Adversarial loss. We use the traditional adversarial loss as presented in [14]. Given the front view normal and depth maps $\mathbf{N}_{f}$ and $\mathbf{D}_{f}$, the reflected back view maps $\mathbf{N}_{b}^{\prime}$ and $\mathbf{D}_{b}^{\prime}$, we define the adversarial loss as:

$$
\begin{aligned}
& L_{G A N}(G, D)= \\
& \quad \mathbb{E}_{\left(\mathbf{N}_{f}, \mathbf{D}_{f}\right)}\left[\log \left(D\left(\mathbf{N}_{b}, \mathbf{D}_{b}\right)\right)\right]+ \\
& \mathbb{E}_{\left(\mathbf{N}_{f}, \mathbf{D}_{f}, \mathbf{N}^{\prime}{ }_{b}, \mathbf{D}^{\prime}{ }_{b}\right)}\left[\log \left(1-D\left(G\left(\mathbf{N}_{f}, \mathbf{D}_{f}, \mathbf{N}^{\prime}{ }_{b}, \mathbf{D}^{\prime}{ }_{b}\right)\right)\right)\right]
\end{aligned}
$$

Similarity loss. Similar to many existing image-to-image translation tasks, we use L1 loss between the output and ground truth images as a similarity measure for the depth maps. Since the normals represent an orientation, for which deviation is more accurately represented by an angle, we use cosine similarity for the normal maps. Given the predicted back view maps $\hat{\mathbf{N}}_{b}$ and $\hat{\mathbf{D}}_{b}$ produced by the generator and the ground truth normal and depth images $\mathbf{N}_{b}$ and $\mathbf{D}_{b}$ :

$$
\begin{aligned}
L_{d} & =\left\|\mathbf{D}_{b}-\hat{\mathbf{D}}_{b}\right\|_{1} \\
L_{n} & =\left[\mathbf{N}_{b}, \hat{\mathbf{N}}_{b}\right]_{\cos },
\end{aligned}
$$

where

$$
[\mathbf{A}, \mathbf{B}]_{\cos }=\sum_{i, j}\left(1-\frac{\mathbf{A}(i, j) \cdot \mathbf{B}(i, j)}{\|\mathbf{A}(i, j)\| \cdot\|\mathbf{B}(i, j)\|}\right) .
$$

\subsection{Surface Reconstruction}

We fuse the per-pixel positions and normals form the front, reflected, and predicted back maps to generate an oriented point cloud and use screened Poisson surface reconstruction [22] to surface this input. To produce closed meshes we use Dirichlet boundary conditions, and use an interpolation weigth of 4 to promote interpolation of input points. Our fusion process automatically corrects quantization artifacts and inaccuracies in the computed maps 


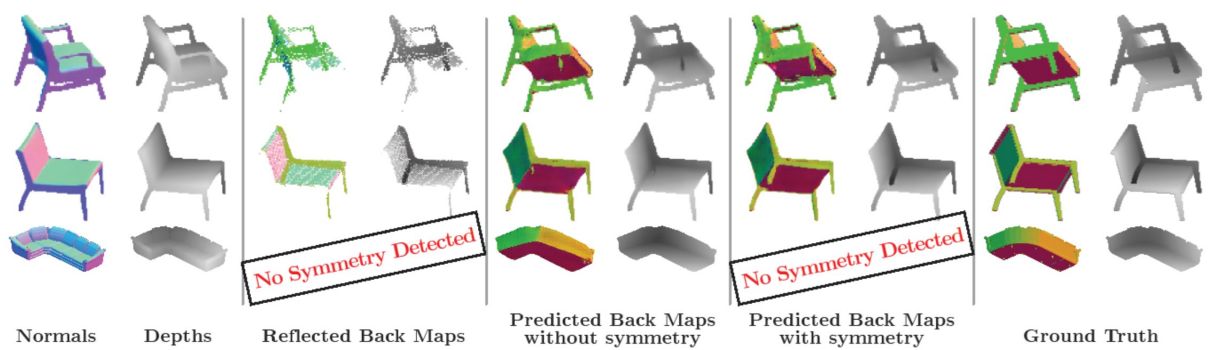

Figure 4: Back map prediction. Given front and reflected depth+normal maps our image-to-image translation network predict the corresponding back view maps; we correctly identify asymmetric models and predict the correspondig back maps usig a separately trained predictor which used only front maps as input.

that can lead to catastrophic reconstruction failures, and accounts for point density assumptions made by typical reconstruction methods.

Fusion. Similar to many other methods, screened Poisson reconstruction [22] expects input points originating from the same surface to be closer to one another than to points originating from an oppositely oriented surface. To satisfy this property given any pair of map points with oppositely oriented normals (one front facing and one back facing) which are within image-space distance of less than two pixels of one another, we ensure that the depth difference between them is at least two pixels, by moving the back facing point backward if needed. Following the visibility prior, we expect the front map points to be more reliable and closer to the viewer than points from other sources, thus we discard reflected and back map points its they are closer to the viewer than front map points at the same $x, y$. Following the same argument, we expect back map points to be farthest from the viewer, however in cases of depth conflicts we trust reflected maps more that back prediction. Thus we discard back map points that are closer to the viewer than reflected map points at the same $x, y$. Lastly, we remove outliers which we classify as points which are above a fixed threshold away from all four of their immediate imagespace neighbors in the same map along the depth axis. The logic behind this criterion is that while we expect the depth maps to exhibit discontinuities, we do expect local features to be larger than one pixel.

More fine grain implementation details are provided in the supplementary material.

\section{Experiments}

We tested our methods on a large body of models across different classesand performed both qualitative and quantitative comparisons, as well as ablation studies demonstrating the impact of the different algorithmic choices made. Additional results, including ones generated from $256 \times 256$ images, are provided in the supplementary material.

Dataset. In our experiments we use the ShapeNet Core dataset [3] and its official training/testing split, which includes 13 object categories. To compare against both methods that use this split and those that use the training/testing split of Choi et al. [7] in the results below we report all quantities on the intersection of the two test sets that none of the models have seen in training. To be comparable, we train our orthographic front view prediction using the training split of the images corresponding to the training models provided by Choi et al. [7] at resolution $137 \times 137$ pixel. We follow [27] and others and use the first, random view, provided by Choi et al. for the test set. To generate both the front and back view maps for training, we rendered orthographic depth and normal map from these views and their opposites. We use the canonical symmetry plane (yz) of the training shapes that are symmetric around it for generating reflected maps for training.

Metrics. For evaluation we use the mesh-to-mesh symmetric $L_{1}$ distance (MD) [8] and Chamfer $L_{1}$ distance (CD) between the ground truth and reconstructed meshes. We measure MD using Metro [8], a well established measurement tool in the geometry processing community, using default Metro parameters. We measure CD using the implementation provided by [27] across $100 \mathrm{~K}$ evenly sampled points on the two meshes. The core difference between these metrics is that Metro looks for closest distance from a sample point on one mesh to any of the triangles on the other, while $\mathrm{CD}$ only considers sample to sample distances and thus is inherently more dependent on sampling density and quality. We report MD using the diagonal of the ground truth bounding box as unit 1 , and follow the convention in [27] when reporting $\mathrm{CD}$.

Implementation Details. We use 'adam' optimizer with learning rate 0.0001 and batch size of 4 for training our orthographic front view predictor. We use the loss and corresponding architecture and parameter settings from [44]. For symmetry detection we use 20 iterations each with $8 \mathrm{~K}$ plane samples when computing initial plane up to 400 iterations for ICP; for correspondence pruning we use thresholds of 4 pixels and $60^{\circ}$ on position and normals. We re- 
ject reflection planes as violating the visual hull threshold, if over $5 \%$ of the reflected pixels are at least 5 pixels outside the silhouette, and reject them as violating visibility if over $15 \%$ of the pixels are in front of the front view. We use 'adam' optimizer with learning rate 0.0002 and batch size of 1 for training our back prediction network, and use 5 random views out of the 24 provided by [7]. For final reconstruction step outlier removal we use threshold of 4 pixel sizes. These parameters are kept constant for all experiments in the paper.

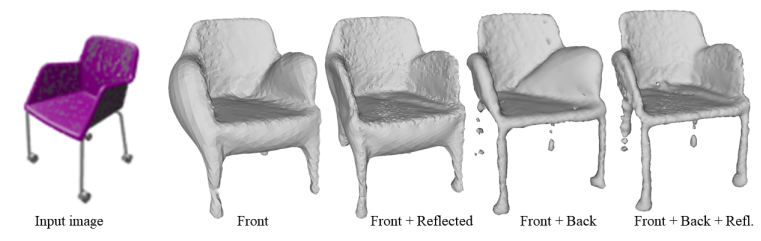

Figure 5: Final reconstruction ablation. Each of the chosen intermediate maps improves the final surface reconstruction quality.

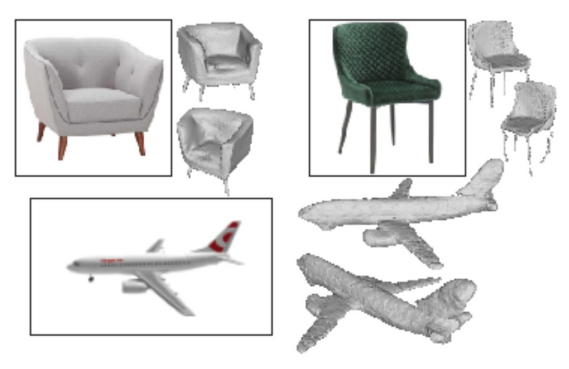

Figure 6: 3D shape from real images. Our results (right) produced from real images (left) While our results are not perfect, they provide reasonable approximation of the shown shape.

\subsection{Assessing Back Map Prediction}

Figure 4 shows the results of our core back view from front view prediction step for several different-class examples. The depth and normal maps clearly convey that our networks can successfully predict the backside of the raw input images, replicating front features when appropriate and consistently completing entirely invisible features such as the back of the sofa or the occluded chair legs.

Back Prediction Ablation. To verify that the symmetry information improves back map prediction, we tested our prediction method with and without the reflected maps. We measure this impact on ground truth front maps of models from the test set to decouple performance of back prediction from the rest of the method. Table 2 shows the impact of using reflected maps on the accuracy of the back prediction for airplanes. We report performance in terms of average $L_{1}$ distance for the depth and average one minus cosine distance for the normal map; effectively this results in Eq 2. As the numbers consistently show the use of reflection maps during prediction is important for the accuracy of resulting back maps. Figure 4, illustrates this difference on real image data. The impact of symmetry is most apparent in the normal maps, where chair legs with different normals are easily recognizable.

\begin{tabular}{lc}
\hline & MD \\
\hline Ours (no symmetry) & 0.0132 \\
Ours (with symmetry) & $\mathbf{0 . 0 1 2 9}$ \\
\hline
\end{tabular}

Table 1: Surface ablation study on airplane models, measuring mesh-to-mesh distance to ground truth on surfacing outputs generated without (top) and with (bottom) reflection maps. Our outputs are closer to ground truth and visually more symmetric (Figure 5).

\begin{tabular}{lcc}
\hline & depth $\left(\operatorname{avg} L_{1}\right)$ & normal $\left(\operatorname{avg}\left[1-\cos \left(a^{\prime}, a\right)\right]\right)$ \\
\hline Ours (no sym) & 0.000593 & 0.00310 \\
Ours (with) & $\mathbf{0 . 0 0 0 5 7 8}$ & $\mathbf{0 . 0 0 2 6 8}$ \\
\hline
\end{tabular}

Table 2: Front map prediction ablation: on airplanes. Ground truth front maps are used with (bottom row) and without (top row) reflected maps. Performance is reported in terms of average per pixel and across model $L_{1}$ and 1 minus cosine distance of depth and normal back predictions respectively. Clearly the use of reflected maps is shown to be beneficial.

3D Reconstruction Ablation. The final surface reconstruction uses as input a union of points from the front, reflected front, and predicted back views. To evaluate the importance of using these maps for reconstruction, we perform an ablation study on chairs and airplanes where we only feed some of the available maps into the surfacing software (Figure 5). Somewhat self-evidently (Figure 5) accurate reconstruction is essentially impossible absent back view information. More interestingly, our measurements (Table 1) show that including the reflected maps in the point cloud used for final reconstruction makes a more subtle but important impact on the quality of the results. The qualitative impact of incorporating the reflected map points is also quite significant (see Figure 5) - human observers expect many man-made objects to be symmetric, and reconstructions that do not respect symmetry appear far less satisfactory from a user perspective. The results incorporating symmetry are thus both better quantitatively and more visually realistic or believable. 

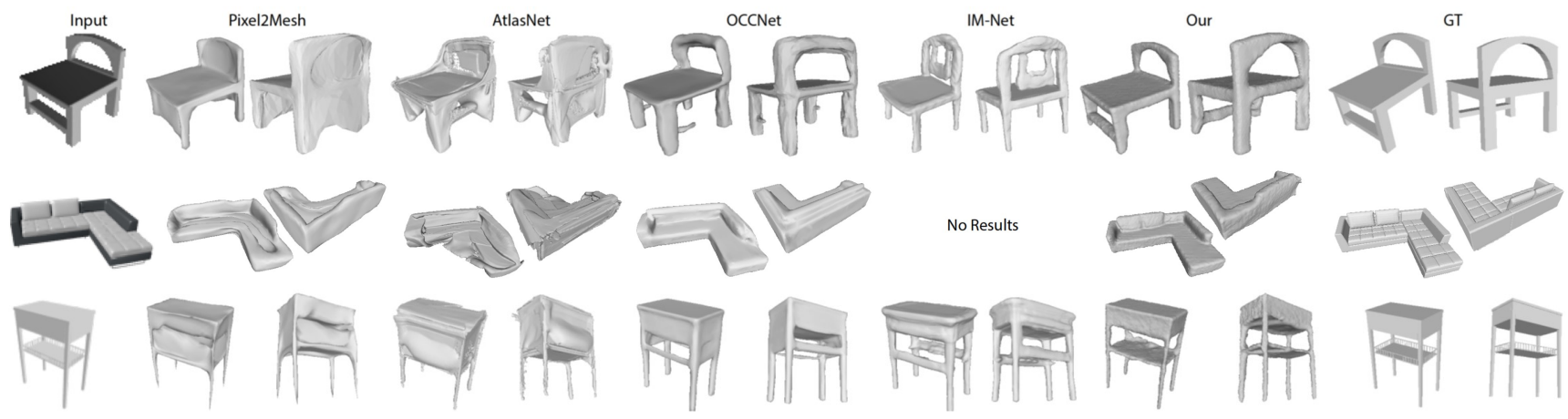

Figure 7: Qualitative comparison to state-of-the-art. Visual comparison of our results and those produced by Pixel2Mesh [38], AtlasNet [16], OccNet [27], and IM-NET [6] . In all examples our results are more consistent with the input images. For some of the methods, e.g. [27, 6] the strong shape priors result in meshes that are close to what the network considers a reasonable object but very far away from the input image.

\begin{tabular}{|c|c|c|c|c|c|c|c|c|c|c|c|c|c|c|c|}
\hline & \multirow[b]{2}{*}{ METHODS } & \multicolumn{13}{|c|}{ CATEGORY } & \multirow[b]{2}{*}{ MEAN } \\
\hline & & 穿 & 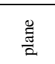 & 总 & $\begin{array}{l}\overline{0} \\
\overline{\bar{u}} \\
\end{array}$ & 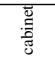 & 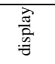 & 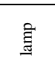 & 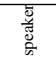 & 童 & 要 & 卷 & 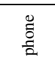 & $\begin{array}{l}\bar{\Xi} \\
\dot{y} \\
\varrho\end{array}$ & \\
\hline \multirow{5}{*}{ 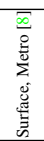 } & Ours & 0.013 & 0.013 & 0.013 & 0.014 & 0.014 & 0.014 & 0.019 & 0.019 & 0.012 & 0.015 & 0.012 & 0.012 & 0.016 & 0.0144 \\
\hline & ONet ( Mescheder et al. [27]) & 0.019 & 0.016 & 0.017 & 0.017 & 0.017 & 0.022 & 0.033 & 0.036 & 0.016 & 0.019 & 0.020 & 0.021 & 0.021 & 0.0213 \\
\hline & AtlasNet ( Groueix et al. [16]) & 0.018 & 0.014 & 0.016 & 0.016 & 0.018 & 0.016 & 0.028 & 0.025 & 0.013 & 0.019 & 0.021 & 0.012 & 0.018 & 0.0181 \\
\hline & Pixel2Mesh ( Wang et al. [38]) & 0.016 & 0.020 & 0.011 & 0.016 & 0.012 & 0.016 & 0.021 & 0.020 & 0.014 & 0.014 & 0.014 & 0.011 & 0.021 & 0.0160 \\
\hline & IM-NET ( Chen et al. [6]) & 0.023 & 0.017 & 0.018 & I & I & I & I & 1 & 0.015 & 1 & 0.029 & I & I & 0.0206 \\
\hline \multirow{5}{*}{ 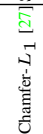 } & Ours & 0.021 & 0.017 & 0.019 & 0.021 & 0.023 & 0.020 & 0.023 & 0.027 & 0.015 & 0.023 & 0.019 & 0.017 & 0.022 & 0.0206 \\
\hline & ONet ( Mescheder et al. [27]) & 0.028 & 0.023 & 0.021 & 0.022 & 0.028 & 0.031 & 0.041 & 0.047 & 0.020 & 0.025 & 0.028 & 0.028 & 0.027 & 0.0283 \\
\hline & AtlasNet ( Groueix et al. [16]) & 0.027 & 0.021 & 0.020 & 0.022 & 0.027 & 0.023 & 0.038 & 0.035 & 0.017 & 0.025 & 0.032 & 0.017 & 0.027 & 0.0254 \\
\hline & Pixel2Mesh ( Wang et al. [38]) & 0.022 & 0.025 & 0.016 & 0.021 & 0.019 & 0.022 & 0.028 & 0.029 & 0.018 & 0.019 & 0.022 & 0.015 & 0.028 & 0.0221 \\
\hline & IM-NET ( Chen et al. [6]) & 0.035 & 0.024 & 0.021 & I & I & l & 1 & 1 & 0.017 & I & 0.043 & 1 & 1 & 0.0280 \\
\hline
\end{tabular}

Table 3: Comparisons against state-of-the-art. We compare our results against Pixel2Mesh [38], AtlasNet [16], OccNet [27], and IM-NET [6] measuring both mesh-to-mesh distance (MD) and $L_{1}$ Chamfer Distance (CD). Our method provides the best results overall for both metrics, outpeorforming the closest competitors on nine out of 13 classes.

\subsection{Single view 3D Shape Reconstruction}

We tested our method on both 137 and 256 resolution images from the ShapeNet dataset [3] as discussed above. Representative results are shown in figures $1,2,8$, and 6. These results show that our method generates high quality results across a large number of classess, and object geometries.

Comparisons. We compare our method to a range of state-of-the-art techniques, including Pixel2Mesh [38], AtlasNet [16], OccNet [27], and IM-NET [6] (Table 3). We use published codes and pre-trained weights from respective papers to reproduce all results. We are unable to provide direct comparison to 3DN [41] because of issues with their published code ${ }^{3}$. Figure 8 shows some representative comparisons. As demonstrated across multiple inputs our method consistently captures fine model details more accurately than other methods. We note that while we use the CD metric from [27] to report the performance, we are recomputing all the numbers. Both [27] and Chen et al. [6] rely on watertight training models, that only approximate ground truth, and report Chanfer distance wrt to these proxies, while we seek to measure distances wrt to the real

\footnotetext{
${ }^{3}$ https://github.com/laughtervv/3DN/issues/4
}

ground truth. Table 3 verifies quantitatively what the preceding qualitative results have shown: Our reconstructions are much closer to the ground truth shapes than those of other learning-based shape reconstruction methods, reducing both MD and CD by as much as $19 \%$ (on cars) and $18 \%$ (on lamps) respectively. On average across the 13 categories we are $12.5 \%$ better than the closes competing method, which turns out to be [38]. Overall our method is more accurate on 9 out of 13 categories; we perform worse on cars, cabinets, phones and sofa where we are only marginally worse than the best method.

Application to real images. Figure 6 shows that, although trained on synthetic renderings, our method yields realistic reconstructions on real images when provided with a segmentation mask or simple background.

\section{Conclusion}

We presented a novel single view 3D reconstruction method anchored around prediction of back view maps from front view information and demonstrated it to improve on the state of the art. Key to the success of our method is a combination of advanced learning approaches with geometry priors that motivate our algorithmic choices. Notably, 
our prediction of 2.5D front maps from images can be potentially substituted by other sources of depth and normal maps, such as depth scanners or sketch processing systems, enabling direct reconstruction of complete 3D objects from such data.

Acknowlegements: This work was funded in part by the Vector Institute for AI, Canada CIFAR AI Chair, NSERC Canada Research Chair (CRC), Compute Canada RRG, and NSERC Discovery Grants.

\section{References}

[1] Dror Aiger, Niloy J. Mitra, and Daniel Cohen-Or. 4-points congruent sets for robust pairwise surface registration. ACM Trans. Graph., 27(3), 2008. 4

[2] Matthew Berger, Andrea Tagliasacchi, Lee Seversky, Pierre Alliez, Joshua Levine, Andrei Sharf, and Claudio Silva. State of the art in surface reconstruction from point clouds. In EUROGRAPHICS star reports, volume 1, pages 161-185, 2014. 3

[3] Angel X. Chang, Thomas A. Funkhouser, Leonidas J. Guibas, Pat Hanrahan, Qi-Xing Huang, Zimo Li, Silvio Savarese, Manolis Savva, Shuran Song, Hao Su, Jianxiong Xiao, Li Yi, and Fisher Yu. Shapenet: An information-rich 3d model repository. CoRR, abs/1512.03012, 2015. 1, 2, 6, 7, 11

[4] Huiwen Chang, Jingwan Lu, Fisher Yu, and Adam Finkelstein. Pairedcyclegan: Asymmetric style transfer for applying and removing makeup. In Proceedings of the IEEE Conference on Computer Vision and Pattern Recognition, pages 40-48, 2018. 3

[5] Xu Chen, Jie Song, and Otmar Hilliges. Monocular neural image based rendering with continuous view control. In Proceedings of the IEEE International Conference on Computer Vision, pages 4090-4100, 2019. 3

[6] Zhiqin Chen and Hao Zhang. Learning implicit fields for generative shape modeling. IEEE Computer Vision and Pattern Recognition (CVPR), 2019. 2, 3, 8, 11, 12, 13

[7] Christopher B Choy, Danfei Xu, JunYoung Gwak, Kevin Chen, and Silvio Savarese. 3d-r2n2: A unified approach for single and multi-view $3 \mathrm{~d}$ object reconstruction. In European conference on computer vision, pages 628-644. Springer, 2016. 2, 6, 7, 11

[8] P. Cignoni, C. Rocchini, and R. Scopigno. Metro: Measuring error on simplified surfaces. Computer Graphics Forum, 1998. 2, 6, 8, 11

[9] Alexey Dosovitskiy, Jost Tobias Springenberg, and Thomas Brox. Learning to generate chairs with convolutional neural networks. In Proceedings of the IEEE Conference on Computer Vision and Pattern Recognition, pages 1538-1546, 2015. 3

[10] David Eigen, Christian Puhrsch, and Rob Fergus. Depth map prediction from a single image using a multi-scale deep network. In Advances in neural information processing systems, pages 2366-2374, 2014. 3
[11] Haoqiang Fan, Hao Su, and Leonidas J. Guibas. A point set generation network for $3 \mathrm{~d}$ object reconstruction from a single image. In 2017 IEEE Conference on Computer Vision and Pattern Recognition, CVPR, pages 2463-2471, 2017. 2, 3

[12] Valentin Gabeur, Jean-Sébastien Franco, Xavier MARTIN, Cordelia Schmid, and Gregory Rogez. Moulding Humans: Non-parametric 3D Human Shape Estimation from Single Images. In ICCV 2019 - International Conference on Computer Vision, pages 1-10, Seoul, South Korea, 2019. 3

[13] R. Girdhar, D.F. Fouhey, M. Rodriguez, and A. Gupta. Learning a predictable and generative vector representation for objects. In $E C C V, 2016.2,3$

[14] Ian Goodfellow, Jean Pouget-Abadie, Mehdi Mirza, Bing $\mathrm{Xu}$, David Warde-Farley, Sherjil Ozair, Aaron Courville, and Yoshua Bengio. Generative adversarial nets. In Advances in neural information processing systems, pages 2672-2680, 2014. 5

[15] Ned Greene. Environment mapping and other applications of world projections. IEEE Computer Graphics and Applications, 6(11):21-29, 1986. 3

[16] Thibault Groueix, Matthew Fisher, Vladimir G. Kim, Bryan Russell, and Mathieu Aubry. AtlasNet: A Papier-Mâché Approach to Learning 3D Surface Generation. In CVPR 2018, 2018. 2, 3, 8, 11, 12, 13

[17] Xiaoguang Han, Zhen Li, Haibin Huang, Evangelos Kalogerakis, and Yizhou Yu. High-resolution shape completion using deep neural networks for global structure and local geometry inference. In Proceedings of the IEEE International Conference on Computer Vision, pages 85-93, 2017. 3

[18] Christian Häne, Shubham Tulsiani, and Jitendra Malik. Hierarchical surface prediction for $3 \mathrm{~d}$ object reconstruction. In 2017 International Conference on 3D Vision (3DV), pages 412-420. IEEE, 2017. 11

[19] Donald D Hoffman. Visual intelligence: how to create what we see. Norton, New York, NY, 2000. 4

[20] Xun Huang, Ming-Yu Liu, Serge Belongie, and Jan Kautz. Multimodal unsupervised image-to-image translation. In Proceedings of the European Conference on Computer Vision (ECCV), pages 172-189, 2018. 3

[21] Phillip Isola, Jun-Yan Zhu, Tinghui Zhou, and Alexei A. Efros. Image-to-image translation with conditional adversarial networks. 2017 IEEE Conference on Computer Vision and Pattern Recognition (CVPR), pages 5967-5976, 2017. $3,5,11$

[22] Michael Kazhdan and Hugues Hoppe. Screened poisson surface reconstruction. ACM Transactions on Graphics (ToG), 32(3):29, 2013. 2, 3, 5

[23] Hsin-Ying Lee, Hung-Yu Tseng, Jia-Bin Huang, Maneesh Singh, and Ming-Hsuan Yang. Diverse image-to-image translation via disentangled representations. In Proceedings of the European Conference on Computer Vision (ECCV), pages 35-51, 2018. 3

[24] Chen-Hsuan Lin, Chen Kong, and Simon Lucey. Learning efficient point cloud generation for dense $3 \mathrm{~d}$ object reconstruction. In Thirty-Second AAAI Conference on Artificial Intelligence, 2018. 3 
[25] Fayao Liu, Chunhua Shen, Guosheng Lin, and Ian Reid. Learning depth from single monocular images using deep convolutional neural fields. IEEE transactions on pattern analysis and machine intelligence, 38(10):2024-2039, 2016. 3

[26] David Marr. Vision: A computational investigation into the human representation and processing of visual information. MIT press. Cambridge, Massachusetts, 1982. 1

[27] Lars Mescheder, Michael Oechsle, Michael Niemeyer, Sebastian Nowozin, and Andreas Geiger. Occupancy networks: Learning $3 \mathrm{~d}$ reconstruction in function space. In Proceedings IEEE Conf. on Computer Vision and Pattern Recognition (CVPR), 2019. 2, 3, 6, 8, 11, 12, 13

[28] Niloy J Mitra, Leonidas J Guibas, and Mark Pauly. Partial and approximate symmetry detection for $3 \mathrm{~d}$ geometry. ACM Transactions on Graphics (TOG), 25(3):560-568, 2006. 4

[29] Ryota Natsume, Shunsuke Saito, Zeng Huang, Weikai Chen, Chongyang Ma, Hao Li, and Shigeo Morishima. SiCloPe: Silhouette-Based Clothed People. In Proc. CVPR. IEEE, June 2019. 3

[30] Jeong Joon Park, Peter Florence, Julian Straub, Richard Newcombe, and Steven Lovegrove. Deepsdf: Learning continuous signed distance functions for shape representation. CoRR, abs/1901.05103, 2019. 3

[31] Zygmunt Pizlo, Yunfeng Li, Tadamasa Sawada, and Robert M. Steinman. Making a Machine That Sees Like Us. Oxford, 2014. 2

[32] Patsorn Sangkloy, Jingwan Lu, Chen Fang, Fisher Yu, and James Hays. Scribbler: Controlling deep image synthesis with sketch and color. In Proceedings of the IEEE Conference on Computer Vision and Pattern Recognition, pages 5400-5409, 2017. 3

[33] Daeyun Shin, Charless C Fowlkes, and Derek Hoiem. Pixels, voxels, and views: A study of shape representations for single view $3 \mathrm{~d}$ object shape prediction. In Proceedings of the IEEE Conference on Computer Vision and Pattern Recognition, pages 3061-3069, 2018. 3

[34] Ayan Sinha, Asim Unmesh, Qixing Huang, and Karthik Ramani. Surfnet: Generating 3d shape surfaces using deep residual networks. In Proceedings of the IEEE conference on computer vision and pattern recognition, pages 6040-6049, 2017. 3

[35] Xingyuan Sun, Jiajun Wu, Xiuming Zhang, Zhoutong Zhang, Chengkai Zhang, Tianfan Xue, Joshua B Tenenbaum, and William T Freeman. Pix3d: Dataset and methods for single-image $3 \mathrm{~d}$ shape modeling. In Proceedings of the IEEE Conference on Computer Vision and Pattern Recognition, pages 2974-2983, 2018. 2

[36] Maxim Tatarchenko*, Stephan R. Richter*, Ren Ranftl, Zhuwen Li, Vladlen Koltun, and Thomas Brox. What do single-view 3d reconstruction networks learn? 2019. 2

[37] Shubham Tulsiani, Tinghui Zhou, Alexei A Efros, and Jitendra Malik. Multi-view supervision for single-view reconstruction via differentiable ray consistency. In $C V P R$, volume 1, page 3, 2017. 2

[38] Nanyang Wang, Yinda Zhang, Zhuwen Li, Yanwei Fu, Wei Liu, and Yu-Gang Jiang. Pixel2mesh: Generating 3d mesh models from single rgb images. In $E C C V, 2018.2,3,8,11$, 12,13

[39] Peng-Shuai Wang, Chun-Yu Sun, Yang Liu, and Xin Tong. Adaptive O-CNN: A Patch-based Deep Representation of 3D Shapes. ACM Transactions on Graphics (SIGGRAPH Asia), 37(6), 2018. 3

[40] Ting-Chun Wang, Ming-Yu Liu, Jun-Yan Zhu, Andrew Tao, Jan Kautz, and Bryan Catanzaro. High-resolution image synthesis and semantic manipulation with conditional gans. 2018 IEEE/CVF Conference on Computer Vision and Pattern Recognition, pages 8798-8807, 2018. 3

[41] Weiyue Wang, Duygu Ceylan, Radomir Mech, and Ulrich Neumann. 3dn: 3d deformation network. In CVPR, 2019. 2, 3,8

[42] Jiajun Wu, Yifan Wang, Tianfan Xue, Xingyuan Sun, Bill Freeman, and Josh Tenenbaum. Marrnet: 3d shape reconstruction via $2.5 \mathrm{~d}$ sketches. In Advances in neural information processing systems, pages 540-550, 2017. 2, 3

[43] Jiajun Wu, Chengkai Zhang, Tianfan Xue, Bill Freeman, and Josh Tenenbaum. Learning a probabilistic latent space of object shapes via $3 d$ generative-adversarial modeling. In D. D. Lee, M. Sugiyama, U. V. Luxburg, I. Guyon, and R. Garnett, editors, Advances in Neural Information Processing Systems 29, pages 82-90. Curran Associates, Inc., 2016. 2

[44] Jiajun Wu, Chengkai Zhang, Xiuming Zhang, Zhoutong Zhang, William T Freeman, and Joshua B Tenenbaum. Learning shape priors for single-view $3 \mathrm{~d}$ completion and reconstruction. In Proceedings of the European Conference on Computer Vision (ECCV), volume 2, page 3, 2018. 2, 3, 6

[45] Xinchen Yan, Jimei Yang, Ersin Yumer, Yijie Guo, and Honglak Lee. Perspective transformer nets: Learning singleview $3 \mathrm{~d}$ object reconstruction without $3 \mathrm{~d}$ supervision. In D. D. Lee, M. Sugiyama, U. V. Luxburg, I. Guyon, and R. Garnett, editors, Advances in Neural Information Processing Systems 29, pages 1696-1704. 2016. 2, 3

[46] Xiuming Zhang, Zhoutong Zhang, Chengkai Zhang, Joshua B. Tenenbaum, William T. Freeman, and Jiajun Wu. Learning to reconstruct shapes from unseen classes. In Advances in neural information processing systems, pages 2366-2374, 2018. 1, 2

[47] Tinghui Zhou, Shubham Tulsiani, Weilun Sun, Jitendra Malik, and Alexei A Efros. View synthesis by appearance flow. In European conference on computer vision, pages 286-301. Springer, 2016. 3

[48] Jun-Yan Zhu, Taesung Park, Phillip Isola, and Alexei A. Efros. Unpaired image-to-image translation using cycleconsistent adversarial networks. 2017 IEEE International Conference on Computer Vision (ICCV), pages 2242-2251, 2017. 3 


\section{Appendices}

In this document, we supply additional evaluation, training, and implementation details, and provide a more detailed ablation study.

\section{A. Additional qualitative results}

We included only few qualitative experiments in the main paper due to space limitation. In Figure 8 we provide additional experiments that illustrate performance on a broader set of classes and compare our results against state-of-the-art alternatives. In all cases we are able to recover more faithful topology and geometry of objects, including local high-fidelity detail. Our results consistently more accurately reflect the ground-truth geometry. Among all methods compared against we come closest in approximating the geometry of the lamps (rows 1,6), and capturing the details of the boats (rows 4,5) and the airplane (row 7). Contrary to Pixel2Mesh [38] we correctly recover the topology of the input models, accurately capturing handles on the display (row 2) and bed (row 3 ) and correctly recovering the chair back (rows 8,9) and table (last row) leg connectivity. Contrary to methods such as OccNet [27] and IM-NET [6] we do not hallucinate non-existent details (see car, row 10, gun row 11, or table, last row).

\section{B. Additional quantitative evaluation}

Due to lack of space, in the main paper we only report Chamfer $L_{1}$ distance (CD) measured using the implementation in [27] and surface-to-surface distance (MD), as computed by Metro [8], as our error metrics. Here we include additional evaluation, with respect to state-of-the-art, based on the Normal Consistency metric introduced by [27]. Results are reported in Table 4. Since we measure consistency, higher numbers indicate better performance, with 1 being the optimum. Our proposed method works better on 8 out of 13 categories and overall. This provides additional evidence that we improve on the previous state-of-the-art performance. Since we measure accuracy directly against unprocessed ShapeNet models, most of which are not watertight, we do not provide IoU measurements, since those are only valid for comparing watertight meshes.

Additional information on experiments. When comparing our results to other methods we encountered two challenges. First, a number of methods, e.g. OccNet [27] and IM-NET [6] that use watertight proxies of ShapeNet models for training, measure result accuracy against these proxies, instead of the originating ShapeNet models. We seek to measure accuracy against the original ShapeNet models. Second, while we followed the original ShapeNet train/test/val split to evaluate our models, other methods $e . g$. Pixel2Mesh [38], AtlasNet [16] use the split provided by
[7]. To resolve both issues we use pre-trained models provided by the authors of the relevant papers and test them on the intersection of the two test sets. This intersection test set contains 1685 models: 197 airplanes, 83 benches, 62 cabinets, 149 cars, 324 chairs, 48 displays, 101 lamps, 63 speakers, 75 rifles, 132 sofas, 339 tables, 46 cellphones, and 66 boats. We then measure all reported metrics on these results, comparing them against the original ground truth ShapeNet [3] models.

Each method we compare to uses a different scaling convention. We apply the transformations used by those methods to transform their outputs accordingly to match ground truth models. For AtlasNet [16], OccNet [27] and Pixel2Mesh [38], we do the exact transformations they described in their papers and supplementary materials. For IM-NET [6] we follow the transformations used in the voxelization process they utilized [18].

\section{Scalability to High Resolution}

Our Front2Back map-based formulation can easily scale to operate at a higher resolutions. To do so, we simply need to train (i) image to front map and (ii) front2back modules to operate at the higher resolution (i.e., take higher resolution image as input and produce, equivalent, higher resolution depth and normal maps as outputs). The rest of our framework can remain exactly as is. To illustrate this beneficial capability, we report additional experiments we didn't have space to include in the main paper. We report performance at $256 \times 256$ resolution on the chair and plane classes. Results are qualitatively illustrated in Figure 9 and quantitatively analyzed in Table 5. Higher resolution maps allow recovery of finer geometric detail observed in the outputs. For example, in our higher-resolution results, the legs on the chairs appear much cleaner and geometrically more accurate (row 2) and planes have much more decernable engines and other fine detail (row 3 and 4). This is quantified in Table 5, which shows significantly improved reconstruction scores (approximately 15\% improvement on average) as compared to our main $137 \times 137$ resolution model.

\section{Implementation Details}

In this section, we add more details for our implementation which is not listed in the paper.

Symmetry plane detection. We represent the symmetry planes as 3-dimensional vectors $(\phi, \theta, d)$, where $\phi, \theta$ are the polar angles of the plane's orientation, and $d$ is the distance of the plane to the origin. We use mean-shift clustering with the Epanechnikov kernel to generate an initial symmetry plane guess.

Neural network architecture. We train pix2pix [21] with the resnet- 9 block architecture to predict the back view ori- 


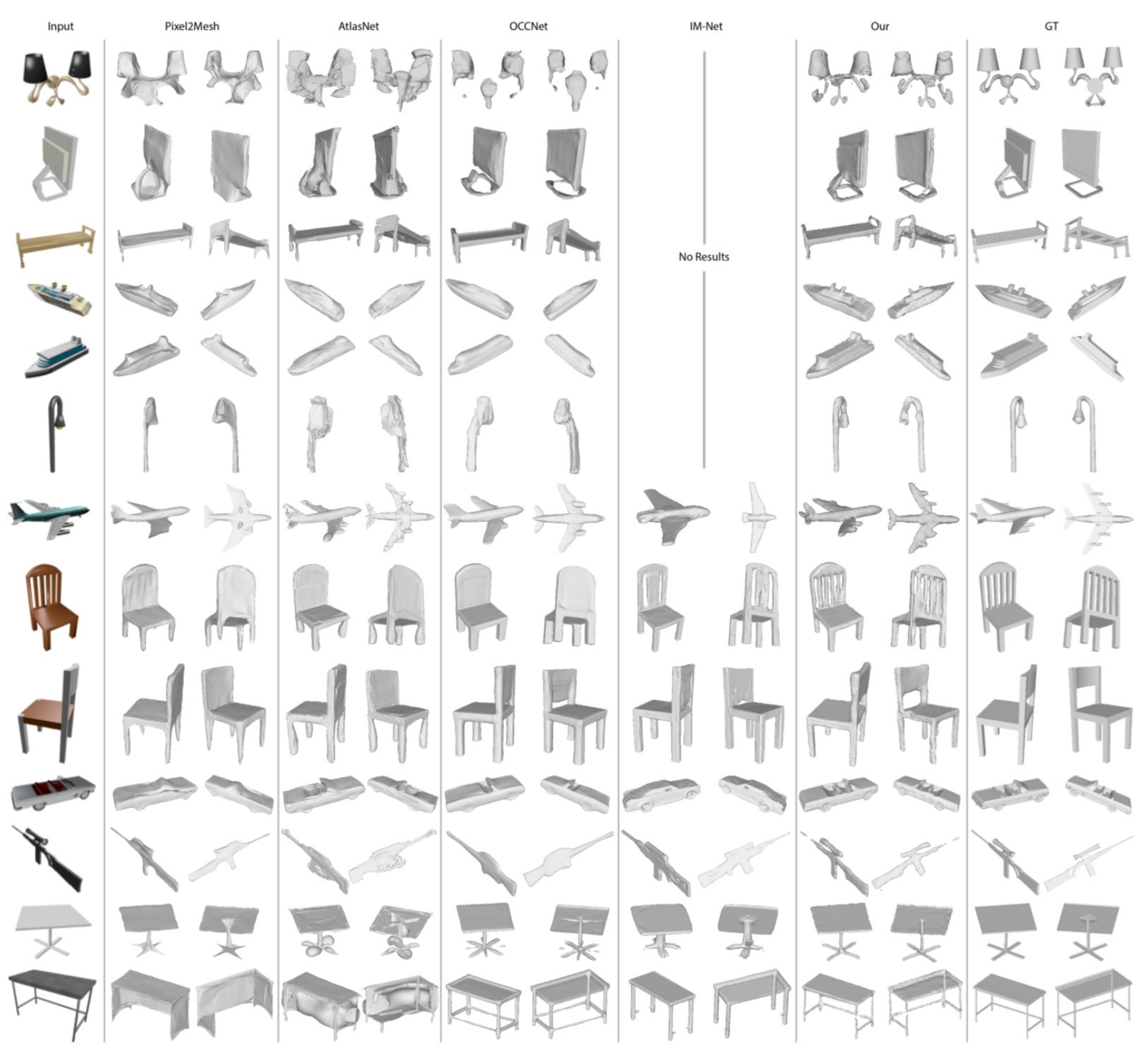

Figure 8: Qualitative comparison to state-of-the-art. Visual comparison of our results and those produced by Pixel2Mesh [38], AtlasNet [16], OccNet [27], and IM-NET [6]. Our results can recover the shape of the input well and capture much more details than others, without halucinating non-existent details. Note that as IM-NET [6] only provides pre-trained models on five classes, we cannot compare with them on other classes such as lamps, displays, etc.

ented depth maps. Our input images are $256 \times 256$ pixels and have eight channels (three channels for the normals, one channel for the depth; both for front view and reflected view). In case of $137 \times 137$ model we pad to 256 ; for the high-resolution model we directly use $256 \times 256$ inputs. For the front normal and depth map prediction we train separate networks for $137 \times 137$ and $256 \times 256$ resolutions.

Training scheme. We train separate models for the image to $2.5 \mathrm{D}$ map representation and the back2front reconstruc- tion. Both networks are trained independently on ground truth data. The learning rate is set to $2 e-4$ and decays by 0.5 after 20 epochs; we train for a total of 40 epochs. Our training data set contains independent classes such as car, airplane, etc.; we train separate models for each class. This is consistent with other papers in the area. 


\begin{tabular}{|c|c|c|c|c|c|c|c|c|c|c|c|c|c|c|}
\hline \multirow[b]{2}{*}{ METHODS } & \multicolumn{13}{|c|}{ CATEGORY } & \multirow[b]{2}{*}{ MEAN } \\
\hline & 慗 & $\frac{\tilde{J}}{2}$ & ฮี & 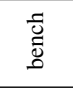 & 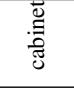 & $\begin{array}{l}\vec{a} \\
\overrightarrow{2} \\
\frac{0}{7}\end{array}$ & 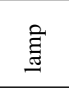 & 峞 & E़ & 焉 & 总 & $\begin{array}{l}\frac{0}{0} \\
\frac{0}{2}\end{array}$ & 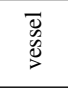 & \\
\hline Ours & 0.771 & 0.759 & 0.734 & 0.674 & 0.763 & 0.821 & 0.734 & 0.780 & 0.672 & 0.781 & 0.789 & 0.856 & 0.733 & 0.759 \\
\hline ONet ( Mescheder et al. [27]) & 0.741 & 0.719 & 0.759 & 0.668 & 0.759 & 0.771 & 0.707 & 0.729 & 0.590 & 0.757 & 0.725 & 0.824 & 0.667 & 0.724 \\
\hline AtlasNet ( Groueix et al. [16]) & 0.701 & 0.682 & 0.725 & 0.627 & 0.725 & 0.772 & 0.641 & 0.738 & 0.549 & 0.734 & 0.671 & 0.839 & 0.614 & 0.694 \\
\hline Pixel2Mesh ( Wang et al. [38]) & 0.741 & 0.747 & 0.698 & 0.685 & 0.782 & 0.837 & 0.705 & 0.767 & 0.636 & 0.780 & 0.762 & 0.881 & 0.681 & 0.746 \\
\hline IM-NET ( Chen et al. [6]) & 0.686 & 0.687 & 0.680 & 1 & 1 & 1 & 1 & 1 & 0.579 & 1 & 0.657 & I & 1 & 0.658 \\
\hline
\end{tabular}

Table 4: Normal Consistency [27] comparisons against state-of-the-art. We compare our results against Pixel2Mesh [38], AtlasNet [16], OccNet [27], and IM-NET [6] measuring Normal Consistency (larger value better). Our method provides the best results overall and outperforms the closest competitors on 8 out of 13 classes.

\begin{tabular}{c|c|cc} 
& METHODS & chair & plane \\
\hline \multirow{2}{*}{ MD } & Ours - 137 & 0.013 & 0.013 \\
& Ours - 256 & $\mathbf{0 . 0 1 1}$ & $\mathbf{0 . 0 1 1}$ \\
\hline \multirow{2}{*}{ CD } & Ours - 137 & 0.021 & 0.017 \\
& Ours - 256 & $\mathbf{0 . 0 1 8}$ & $\mathbf{0 . 0 1 5}$ \\
\hline
\end{tabular}

Table 5: Quantitative results on high resolution $(256 \times 256)$ inputs versus low resolution $(137 \times 137)$ inputs. The performance is increased by $10 \%-20 \%$.

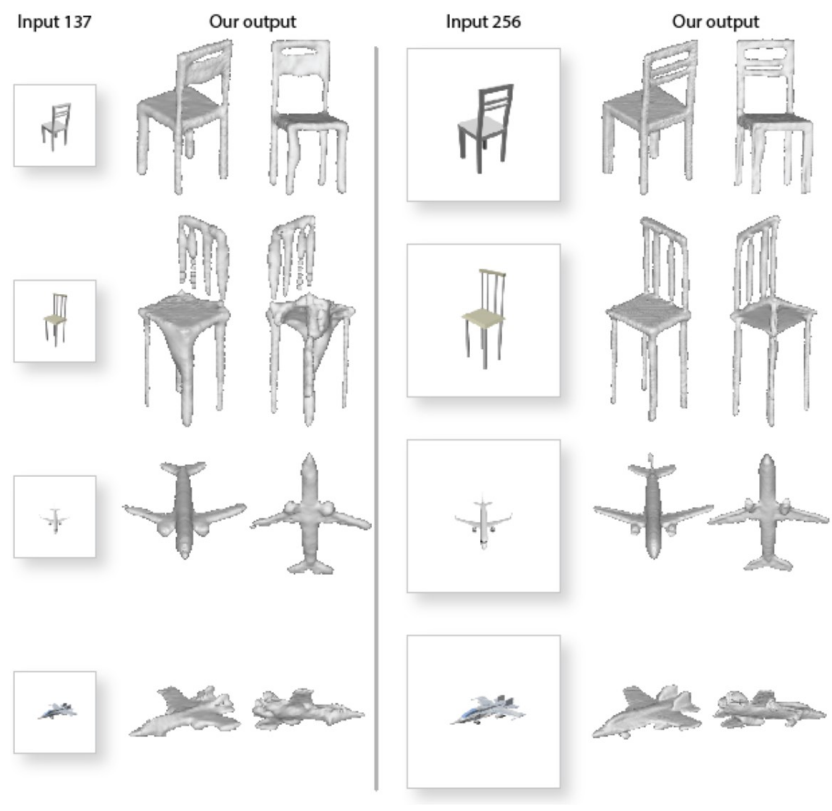

Figure 9: $137 \times 137$ vs $256 \times 256$ resolution results. (left) low resolution images and results. (right) similar view and render style $256 \times 256$ images and corresponding results. The quality of our reconstruction predictably improves with increase in image resolution.

\section{E. Limitations.}

Our method performs well on typical views of everyday objects, where the front and back views (combined with the reflection of the front) describe a big portion of the shape. Predictably, its performance declines on accidental views where the union of these information sources is insufficient to describe the surface (see Figure 10 a). A possible solution for such scenarios is to perform surface completion, e.g. using the method of [27]; or to leverage our existing algorithm rendering the incomplete output from a different direction (ideally one maximally opposite to the incomplete data); repeating the back prediction step, and performing reconstruction using a union of previous and new maps. Our framework does not hallucinate geometry not evident from the images, thus given fuzzy, ambiguous images it is not able to produce meaningful reconstructions (Figure $10 \mathrm{~b}$ ). Symmetry computation on raw images or predicted front maps is a challenging problem; incorrect symmetry plane estimation can potentially lead to reconstruction failures (see Figure $10 \mathrm{c}$ ). We minimize the likelihood of such failures by using very conservative symmetry detection criteria; more robust detection of symmetry planes on image inputs is an interesting future research topic.

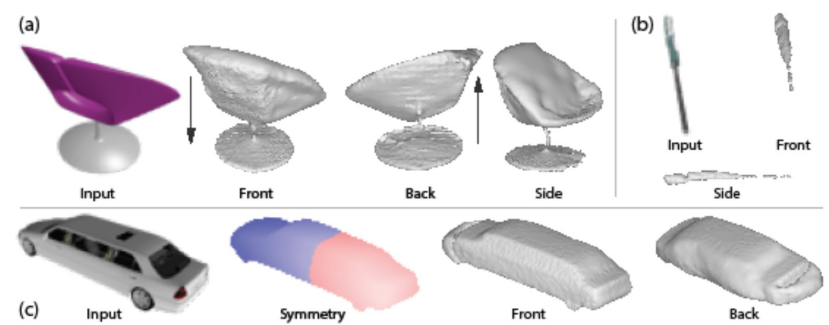

Figure 10: Limitations. (a) Our method's performance declines on accidental view images, such as these, where important details are not discernible from front or predicted back views. (b) Our method cannot recover fuzzy details from ambiguous low-resolution inputs. (c) A wrongly detected reflective symmetry (here left/right instead of front/back) can lead to reconstruction artifacts. 